Haitlinger, R., Šundić, M. (2020): Cooremania kietaensis sp. nov. (Astigmata: Canestriniidae) from Bougainville Island, Papua New Guinea. Agriculture and Forestry, 66 (3): 155-168

DOI: 10.17707/AgricultForest.66.3.13

Ryszard HAITLINGER ${ }^{1}$, Miloje ŠUNDIĆ ${ }^{2}$

\title{
COOREMANIA KIETAENSIS SP. NOV. (ASTIGMATA: CANESTRINIIDAE) FROM BOUGAINVILLE ISLAND, PAPUA NEW GUINEA
}

\section{SUMMARY}

Cooremania kietaensis sp. nov. from Papua New Guinea is described and illustrated, based on males, females and protonymphs. All specimens were obtained from undetermined Lucanidae (Coleoptera). All canestriniids known from Papua New Guinea are listed.

Keywords: Acari, taxonomy, new species, Lucanidae, Coleoptera, Papua New Guinea canestriniids.

\section{INTRODUCTION}

Insects constitute the most diverse form of animal life in terrestrial ecosystems (Bolu, 2016). The family Lucanidae (Coleoptera) includes about 1200 species in the world fauna which occurring mainly in subtropical regions. The canestriniid mites were found only on 47 lucaniid species and some undetermined species, 17 canestriniid species found on Lucanidae [without determined to the genus for example mentioned by Okabe and Goka (2008)] belonging to 9 genera: Amboniophela Haitlinger, 1993, Canestrinia Berlese, 1881, Cooremania Nesbitt, 1976, Haitlingeria Kim, Lee, Choi, Sim and Kim, 2006, Noemiphela Haitlinger, 1991, Rugoniphela Haitlinger, 1991, Sandrophela Haitlinger, 1990, Uriophela Haitlinger, 1991 and Vereoxia Haitlinger, 1995 (Berlese, 1881, Nesbitt, 1976, Haitlinger, 1990a, 1991, 1993, 1995, Kim et al., 2006).

One species of Coleopterophagus (Kishida, 1924) found on Lucanidae not belongs to this genus. Canestriniid mites were found on Lucanidae only in Asia, Europe and Papua New Guinea (Berlese, 1881, Kishida, 1924, Nesbitt, 1976, Haitlinger, 1989a, b, 1990a, 1993, 1995, Kim et al., 2006, Okabe and Goka 2008). From Papua New Guinea were known only two species associated with Lucanidae: Cooremania wauensis Nesbitt, 1976 and Amboinophela bernae Haitlinger, 1993 (Nesbitt, 1976, Haitlinger, 1993). Moreover, in Papua New

\footnotetext{
${ }^{1}$ Ryszard Haitlinger, (corresponding author: ryszard.haitlinger@upwr.edu.pl), Institute of Biology, Invertebrate Systematics and Ecology, Wroclaw University of Environmental and Life Sciences, Kozuchowska 5B, 51-631 Wroclaw, POLAND.

${ }^{2}$ Miloje Šundić, (miloje.sundic@gmail.com) Department of Biology, Faculty of Sciences, University of Montenegro, Cetinjski put b. b. 80000 Podgorica, MONTENEGRO.

Notes: The authors declare that they have no conflicts of interest. Authorship Form signed online.

Received:24/06/2020

Accepted:02/08/2020
} 
Guinea were found 20 other species: Albinorattia igori Haitlinger, 1989 associated with Cetoniinae (Scarabaeidae), Sandrophela kokodaica Haitlinger, 1990 associated with Passandridae, Athogavia arrybasi Haitlinger, 1989, Aurilossongia bolanica Haitlinger, 1989, A. guarana Haitlinger, 1989, A. norae Haitlinger, 1989, Mossongia bissaina Haitlinger, 1989, M. savina Haitlinger, 1989 associated with Dynastinae (Scarabaeidae), Apalotacarus cidaris Summers \& Schuster, 1981, A. fusulus Summers \& Schuster, 1981, A. petilus Summers \& Schuster, 1981, A. rigescens Summers \& Schuster, 1981, Arraphosoma ninax Summers \& Schuster, 1981, Jullongia izae Haitlinger, 1990, Lidiophela pecki (Nesbitt, 1976), Passalophagus georlei Nesbitt, 1976, Phaleratus fentoni Nesbitt, 1976, Photia howdeni Nesbitt, 1976 and Sajanophela alfredae Haitlinger, 1989 associated with Passalidae (Nesbitt, 1976, Summers \& Schuster, 1981a, b, Haitlinger, 1989a, b, 1990a, b).

The genus Cooremania Nesbitt, 1976 is known only from one species found in Papua New Guinea and differing from all other genera by the presence of peg-like setae on ventral part of idiosoma and legs I-III (in all instars). In this paper we describe a new species Cooremania kietaensis from Bougainville Island, Papua New Guinea. It is second species of the genus Cooremania.

\section{MATERIALS AND METHODS}

Three males, two females and three protonymphs of the new species were obtained from the Lucanidae collection of the Museum of Natural History, Wrocław University. All specimens were preserved in $75 \%$ ethanol. Mite specimens were cleared in Nesbitt's solution and mounted in Berlese's medium. Figures were drawn using a Carl Zeiss Axioscope A1 compound microscope. Measurements were made using a NIKON Eclipse 50i compound microscope. All measurements are given in micrometers. The terminology and abbreviations follow Grandjean (1939), Griffiths et al., (1990), Norton (1998), Trach and Khaustov (2011) and Haitlinger and Šundić (2016).

Canestriniidae Berlese, 1884

Cooremania Nesbitt, 1976

Type species: Cooremania wauensis Nesbitt, 1976, by original designation.

Diagnosis. Propodosomal and sejugal structure absent. The presence of peg-like setae on the ventral surface of all stages. Part of setae on legs I-III peglike. Males with well developed adanal suckers.

Cooremania kietaensis sp. nov.

(Figs. 1-20)

Diagnosis. Setae $2 \mathrm{a}, \mathrm{c}_{3}, 3 \mathrm{a}, 4 \mathrm{~b}, \mathrm{ad}_{2}$ and $\mathrm{g}$ peg-like. Setae $\mathrm{p}_{1}, \mathrm{p}_{2}, \mathrm{p}_{3}$ and $\mathrm{h}_{3}$ tubercle-like in males, peg-like in females, setae vF I, vF II and $\mathrm{nG}$ peg-like.

Description. Male (holotype) $(n=3)$ - Idiosoma with ornamentation on propodosoma and anterior part of hysterosoma; Propodosoma with setae vi, si 
and long setae se. Hysterosoma with relatively short setae $\mathrm{c}_{1}, \mathrm{c}_{2}, \mathrm{~d}_{1}, \mathrm{~d}_{2}, \mathrm{e}_{1}, \mathrm{e}_{2}$ and $\mathrm{f}_{2}$ (Fig. 1).

Among setae placed in middle of idiosoma distance $c_{1}-c_{1}$ is the longest, distance $d_{1}-d_{1}$ is longer than distance $e_{1}-e_{1}$ and $e_{2}-e_{2}$ (Table 1).

Table 1. Metric data of Cooremania kietaensis sp. nov.; H - holotype, $\mathrm{P}-$ paratype, $\mathrm{PR}$ - protonymphs.

\begin{tabular}{|c|c|c|c|c|c|c|c|c|}
\hline Character & $\mathrm{H} \mathrm{o}^{-1}$ & $\mathrm{P} \hat{\mathrm{O}}$ & $\bar{P} 0^{1}$ & $\mathrm{P}$ ㅇ & $\mathrm{P}$ 웅 & PR & PR & PR \\
\hline IL & 526 & 480 & 595 & 730 & 677 & 407 & 481 & 430 \\
\hline IW & 440 & 444 & 488 & 666 & 617 & 328 & 385 & 388 \\
\hline GL & 108 & 91 & 100 & 110 & 106 & 90 & 87 & 97 \\
\hline GW & 80 & 74 & 93 & 79 & 73 & 49 & 55 & 64 \\
\hline $\mathrm{c}_{1}$ & 42 & 36 & 40 & 65 & 61 & 42 & 54 & 53 \\
\hline$c_{2}$ & 52 & 46 & 66 & 69 & 69 & 51 & 57 & 65 \\
\hline $\mathrm{c}_{3}$ & 11 & 13 & 14 & 15 & 17 & 12 & 13 & 12 \\
\hline $\mathrm{d}_{1}$ & 36 & 33 & 42 & 66 & 69 & 44 & 44 & 47 \\
\hline $\mathrm{d}_{2}$ & 39 & 57 & 58 & 80 & 76 & 61 & 57 & 68 \\
\hline $\mathrm{e}_{1}$ & 26 & 26 & 27 & 87 & 85 & 47 & - & 55 \\
\hline $\mathrm{e}_{2}$ & 24 & 26 & 31 & 61 & 67 & 51 & 44 & 54 \\
\hline $\mathrm{h}_{1}$ & 500 & 447 & 539 & 577 & - & - & - & - \\
\hline $\mathrm{h}_{2}$ & 472 & 486 & 520 & 517 & 513 & - & 197 & - \\
\hline $\mathrm{h}_{3}$ & 38 & 30 & 27 & 17 & 18 & 12 & 12 & 12 \\
\hline $\mathrm{cp}$ & 425 & 409 & 435 & 490 & 523 & - & - & 243 \\
\hline se & 390 & - & 425 & 498 & - & 310 & - & 391 \\
\hline si & 54 & 63 & 77 & 75 & 73 & 56 & 52 & 52 \\
\hline vi & 62 & 60 & 76 & 75 & 92 & 55 & 71 & 60 \\
\hline SW & 34 & 36 & 44 & - & - & - & - & - \\
\hline SS & 54 & 50 & 66 & - & - & - & - & - \\
\hline ANL & 61 & 58 & 69 & - & - & - & - & - \\
\hline PL & 69 & 60 & 72 & - & - & - & - & - \\
\hline $\mathrm{p}_{1}$ & 37 & 36 & 32 & 16 & 12 & 15 & 12 & 12 \\
\hline $\mathrm{p}_{2}$ & 32 & 30 & 27 & 18 & 13 & 12 & 12 & 12 \\
\hline $\mathrm{p}_{3}$ & 23 & 22 & 23 & 13 & 14 & 18 & 18 & 16 \\
\hline $1 \mathrm{a}$ & 27 & 30 & 30 & 40 & 40 & 31 & 32 & 26 \\
\hline $2 a$ & 15 & 15 & 15 & 16 & 16 & 11 & 13 & 15 \\
\hline $3 a$ & 14 & 13 & 13 & 17 & 16 & 12 & 14 & 15 \\
\hline $4 a$ & 29 & 29 & 36 & 13 & 18 & 19 & - & - \\
\hline $4 b$ & 15 & 14 & 18 & 16 & 15 & - & - & - \\
\hline $\mathrm{g}$ & 11 & 10 & 13 & 19 & 19 & 11 & - & - \\
\hline $\mathrm{f}_{2}$ & 26 & 27 & 32 & 46 & 47 & & & \\
\hline Ta I & 62 & 63 & 68 & 71 & 67 & 44 & 56 & 45 \\
\hline Ta II & 56 & 56 & 64 & 66 & 67 & 49 & 50 & 50 \\
\hline Ta III & 85 & 83 & 83 & 96 & 86 & 65 & 72 & 66 \\
\hline Ta IV & 93 & 93 & 88 & 119 & 112 & 80 & 87 & - \\
\hline Leg I & 221 & 195 & 222 & 227 & 210 & 137 & 157 & 160 \\
\hline Leg II & 200 & 187 & 223 & - & 210 & 151 & 160 & 159 \\
\hline Leg III & 235 & 239 & 250 & 264 & 258 & 198 & 192 & 200 \\
\hline Leg IV & 261 & 229 & 262 & 304 & 282 & 179 & 192 & - \\
\hline Ta I $\omega_{1}$ & 42 & 45 & 55 & 55 & 55 & 35 & 34 & 37 \\
\hline Ta I $\omega_{2}$ & 11 & 11 & 12 & 15 & 12 & 12 & 10 & 18 \\
\hline
\end{tabular}




\begin{tabular}{|c|c|c|c|c|c|c|c|c|}
\hline $\mathrm{Ta} \mathrm{I} \omega_{3}$ & 78 & 66 & 83 & 71 & 82 & 50 & 59 & 60 \\
\hline Ta I d & 95 & 81 & 100 & 92 & 100 & 79 & 88 & 71 \\
\hline Ta I e & 40 & 45 & 46 & 46 & 40 & 33 & 35 & 32 \\
\hline Ta I wa & 40 & 43 & 29 & 47 & 32 & 33 & 27 & 29 \\
\hline Ta I p & 20 & 20 & 20 & 30 & 23 & 16 & 20 & 26 \\
\hline $\mathrm{Ta} \mathrm{I} \mathrm{q}$ & 21 & - & 27 & 44 & 17 & 25 & 27 & 22 \\
\hline Ta I la & 15 & 17 & 22 & 22 & 15 & 16 & 16 & 18 \\
\hline $\operatorname{Ti} \mathrm{I} \varphi$ & 151 & 152 & - & 172 & 171 & 120 & - & 126 \\
\hline Ge I $\sigma$ & 49 & 45 & 51 & 53 & 54 & 40 & 34 & 41 \\
\hline Ge I mG & 26 & 25 & 33 & 31 & 26 & 18 & 24 & 20 \\
\hline Ge I cG & 31 & 34 & 38 & 39 & 36 & 31 & 40 & 35 \\
\hline Fe I vF & 7 & 9 & 8 & 9 & 11 & 11 & 9 & 11 \\
\hline Tr I vTr & 35 & 43 & 37 & 22 & 24 & 32 & 33 & 31 \\
\hline Ta II $\omega$ & 65 & 59 & 56 & 63 & 67 & 51 & 51 & 52 \\
\hline Ta II d & 72 & 98 & 124 & 115 & 105 & 70 & 76 & 66 \\
\hline Ta II e & 36 & 48 & 54 & 55 & 49 & 36 & 29 & 31 \\
\hline Ta II wa & 29 & 44 & 46 & 39 & 35 & 28 & 23 & 28 \\
\hline Ta II ra & 28 & 45 & 38 & - & 44 & 37 & 38 & 21 \\
\hline Ta II la & 16 & 20 & 18 & 18 & 20 & - & - & - \\
\hline Ti II $\varphi$ & 73 & 70 & 83 & 79 & 82 & 55 & 61 & 69 \\
\hline Ge II $\sigma$ & 36 & 33 & 39 & 36 & 37 & 30 & 29 & - \\
\hline Ge II mG & 33 & 25 & 37 & 37 & 32 & 18 & 20 & 22 \\
\hline Ge II cG & 23 & 20 & 20 & 23 & 24 & 24 & 15 & 18 \\
\hline Fe II vF & 10 & 14 & 10 & 12 & 10 & 8 & 10 & 12 \\
\hline Tr II vTr & 35 & 27 & 20 & 30 & 36 & 29 & - & - \\
\hline Ta III d & 48 & 67 & 57 & 127 & 117 & 84 & 73 & - \\
\hline Ta III e & 45 & 62 & 47 & 72 & 70 & 33 & 44 & 48 \\
\hline Ta III w & 25 & 30 & 28 & 54 & 48 & 34 & 28 & 31 \\
\hline Ti III $\varphi$ & 69 & 64 & 83 & 81 & 76 & 44 & 45 & - \\
\hline Ge III nG & 12 & 12 & 12 & 16 & 13 & 12 & 10 & 11 \\
\hline Tr III vTr & 12 & 12 & - & 10 & 13 & 13 & 10 & 13 \\
\hline Ta IV d & 121 & 111 & - & 142 & 134 & 78 & 90 & - \\
\hline Ta IV w & 41 & 38 & 41 & 58 & 40 & 34 & 36 & - \\
\hline Ta IV e & 55 & 60 & - & 75 & 73 & 31 & 34 & - \\
\hline Ti IV $\varphi$ & 102 & - & 121 & 71 & 68 & 33 & 36 & - \\
\hline Tr IV vTr & 48 & 49 & - & 15 & 13 & - & - & - \\
\hline $\mathrm{c}_{1}-\mathrm{c}_{1}$ & 214 & 200 & - & 258 & 222 & 158 & 186 & 198 \\
\hline $\mathrm{d}_{1}-\mathrm{d}_{1}$ & 100 & 101 & 116 & 128 & 121 & 85 & 82 & 108 \\
\hline$e_{1}-e_{1}$ & 126 & 116 & 129 & 132 & 151 & 103 & - & 101 \\
\hline $\mathrm{e}_{2}-\mathrm{e}_{2}$ & 107 & 70 & 106 & 90 & 127 & 75 & - & 58 \\
\hline $\mathrm{ad}_{1}$ & 24 & 22 & 23 & 54 & 57 & - & - & - \\
\hline $\mathrm{ad}_{2}$ & 11 & 10 & 11 & 48 & 55 & - & - & - \\
\hline
\end{tabular}

Ventral side of idiosoma with long setae $\mathrm{cp}, \mathrm{h}_{1}, \mathrm{~h}_{2}$, thin setae $1 \mathrm{a}, 4 \mathrm{a}$, peglike setae $2 \mathrm{a}, \mathrm{c} 3,3 \mathrm{a}, 4 \mathrm{~b}, \mathrm{ad}_{2}$ and $\mathrm{g}$ and tubercle-like setae $\mathrm{p}_{1}, \mathrm{p}_{2}, \mathrm{p}_{3}$ and $\mathrm{h}_{3}$. Apodemes I joint medially to form $\mathrm{Y}$ - shaped structure, other apodemes as in Fig. 2. Aedagus short. The adanal suckers well developed (Fig. 2). 


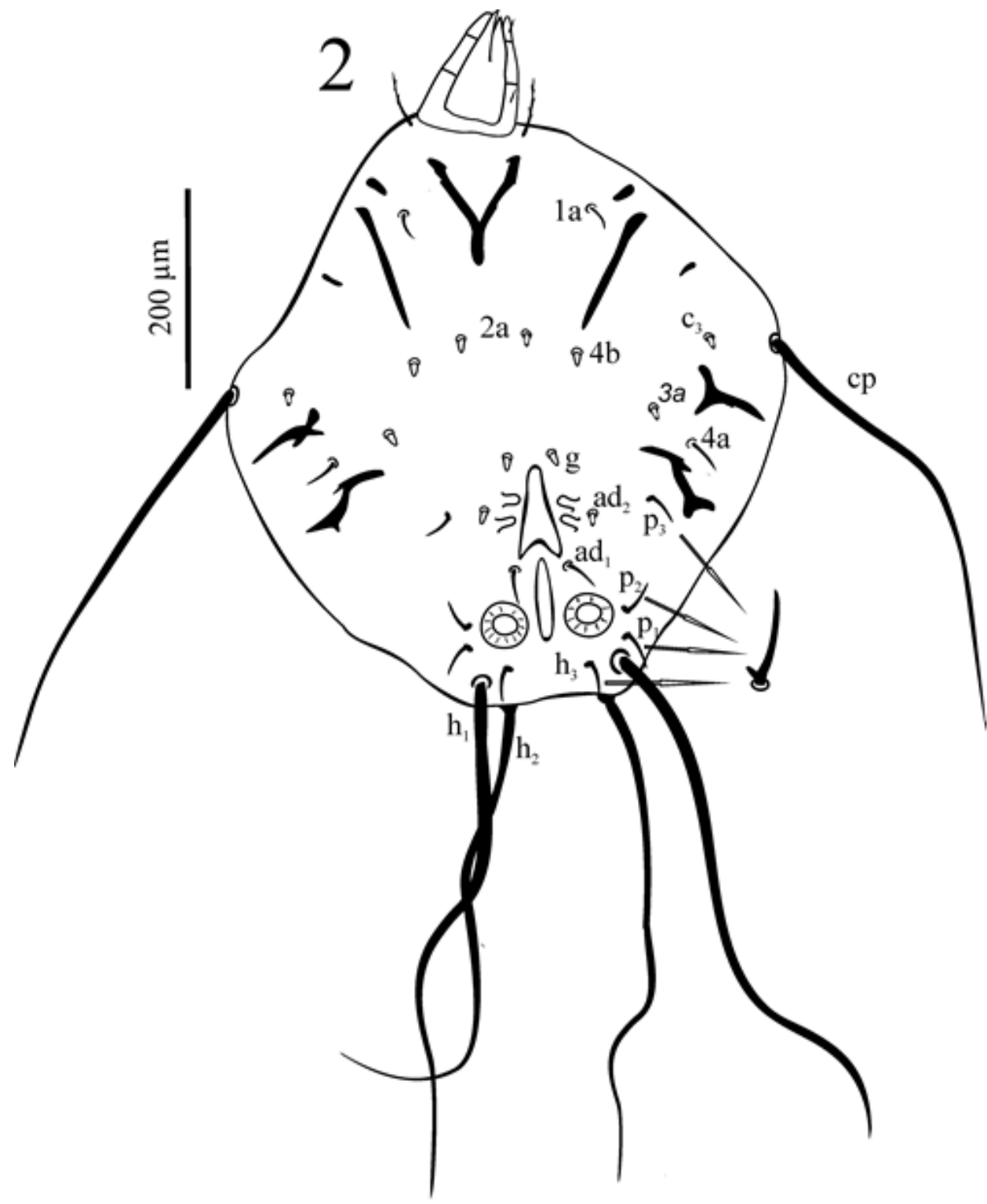

Figures 2. Cooremania kietaensis sp. nov. (holotype-male). Ventral view of idiosoma and gnathosoma. 
Gnathosoma partly covered by idiosoma, subcapitulum with 2 pairs of nude setae, palps with placed terminally solenidion $\omega$ and two pairs of short setae (Fig. 3). Chelicerae with denticles (Fig. 4).
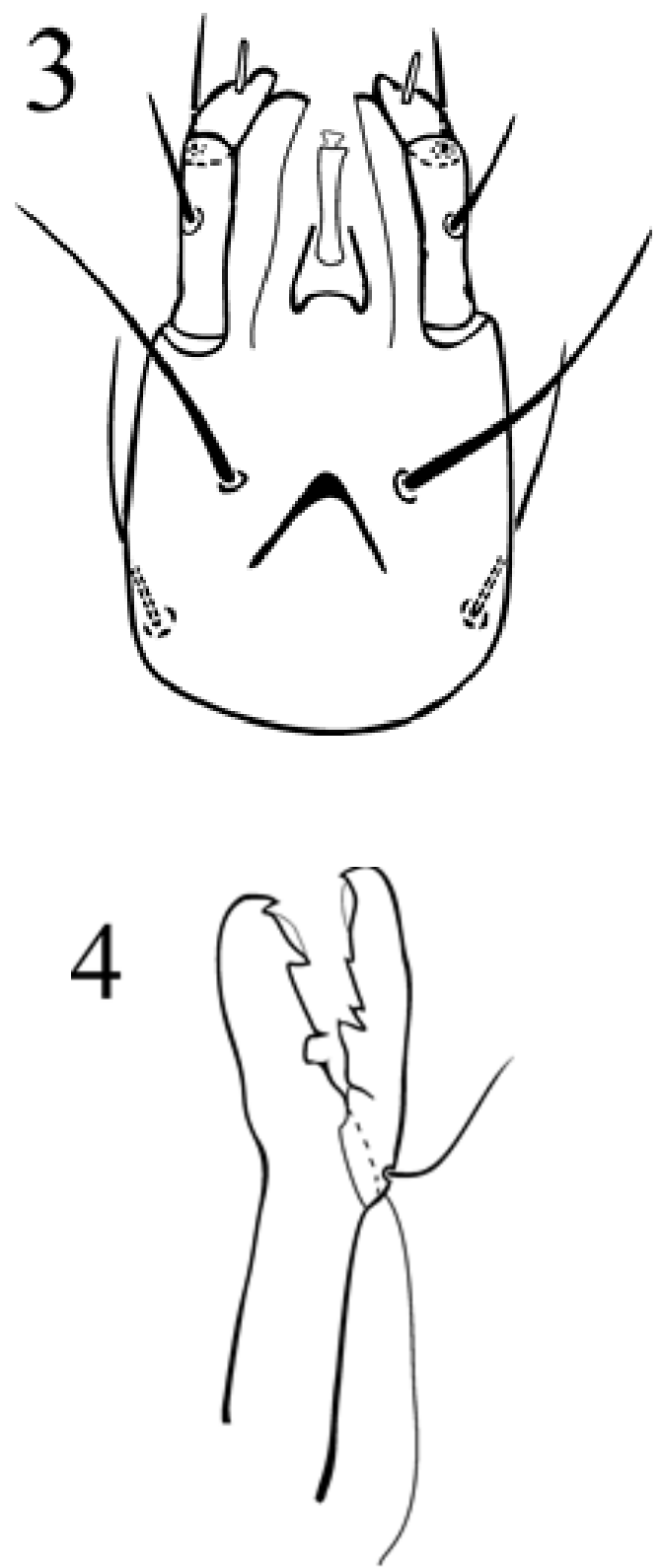

Figures 3-4. Cooremania kietaensis sp. nov. (holotype-male). 3. Gnathosoma, 4. Chelicerae. 

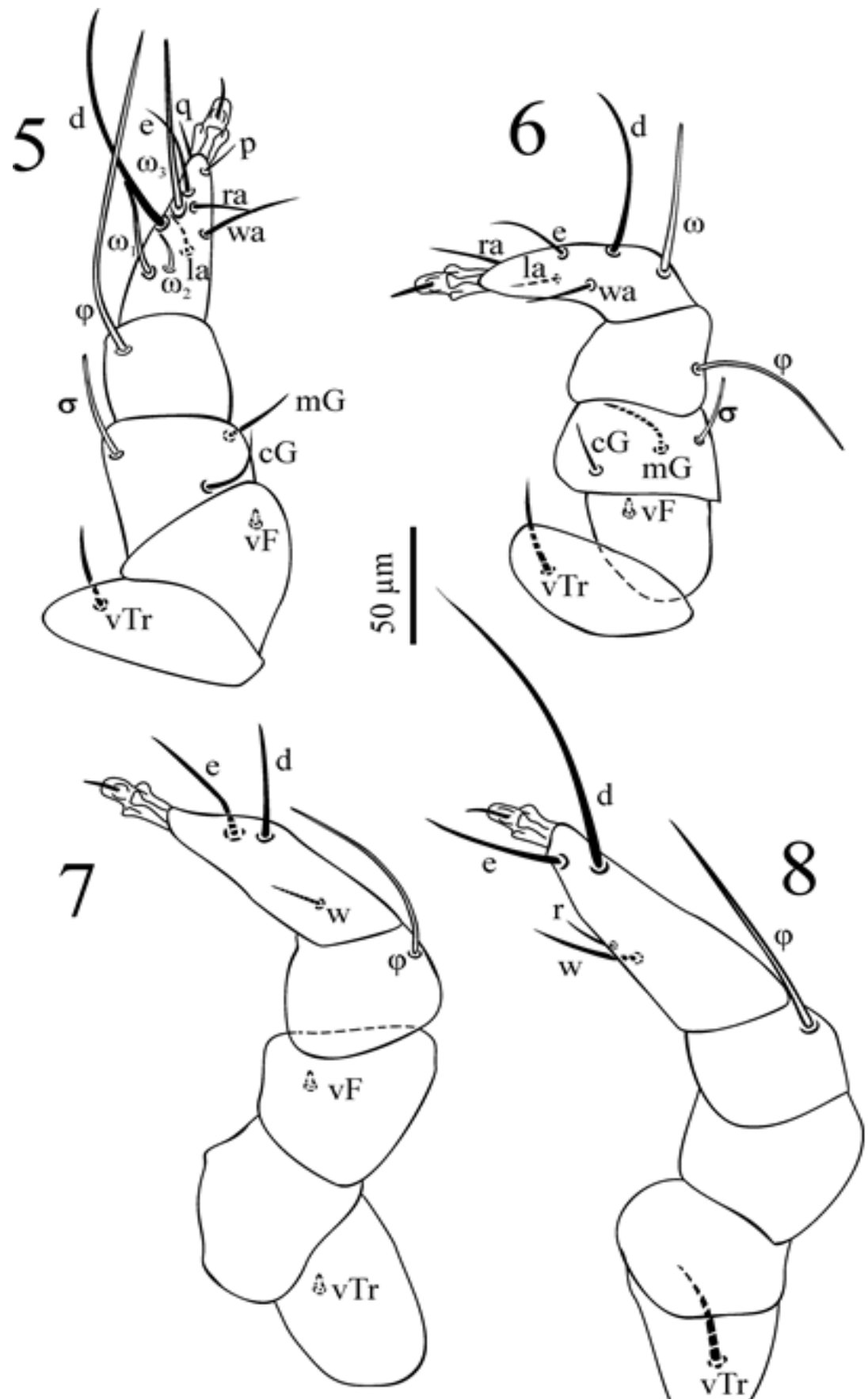

Figures 5-8. Cooremania kietaensis sp. nov. (holotype-male). 5, Leg I, 6, Leg II, 7, Leg III, 8, Leg IV. 
Leg setal formula: Leg I: Ta $-\omega_{1}, \omega_{2}, \omega_{3}$, d, e, q, p, la, ra, wa, s; Ti $-\varphi$, Ge $\sigma, \mathrm{cG}, \mathrm{mG} ; \mathrm{Fe}-\mathrm{vF}$; Tr - vTr (Fig. 5, Table 1). Leg II: Ta $-\omega$, d, e, ra, la, wa, s; $\mathrm{Ti}-\varphi, \mathrm{Ge}-\sigma, \mathrm{mG}, \mathrm{cG} ; \mathrm{Fe}-\mathrm{vF}$; Tr - vTr (Fig. 6). Leg III: Ta $-\mathrm{d}$, e, w, s; Ti $-\varphi$; $\mathrm{Ge}-\mathrm{nG}$; Tr - vTr (Fig. 7). Leg IV: Ta - d, e, w, s; Ti - $\varphi$; Tr - vTr (Fig. 8). Leg I: seta $d$ is the longest on Ta, $\varphi$ is two times longer than $\varphi$ on Ti II and III and distinctly longer than $\varphi$ on Ti IV, seta $v F$ is peg-like. Setae $v F$ on Fe II, seta $\mathrm{nG}$ on Ge III and Tr III are peg-like.
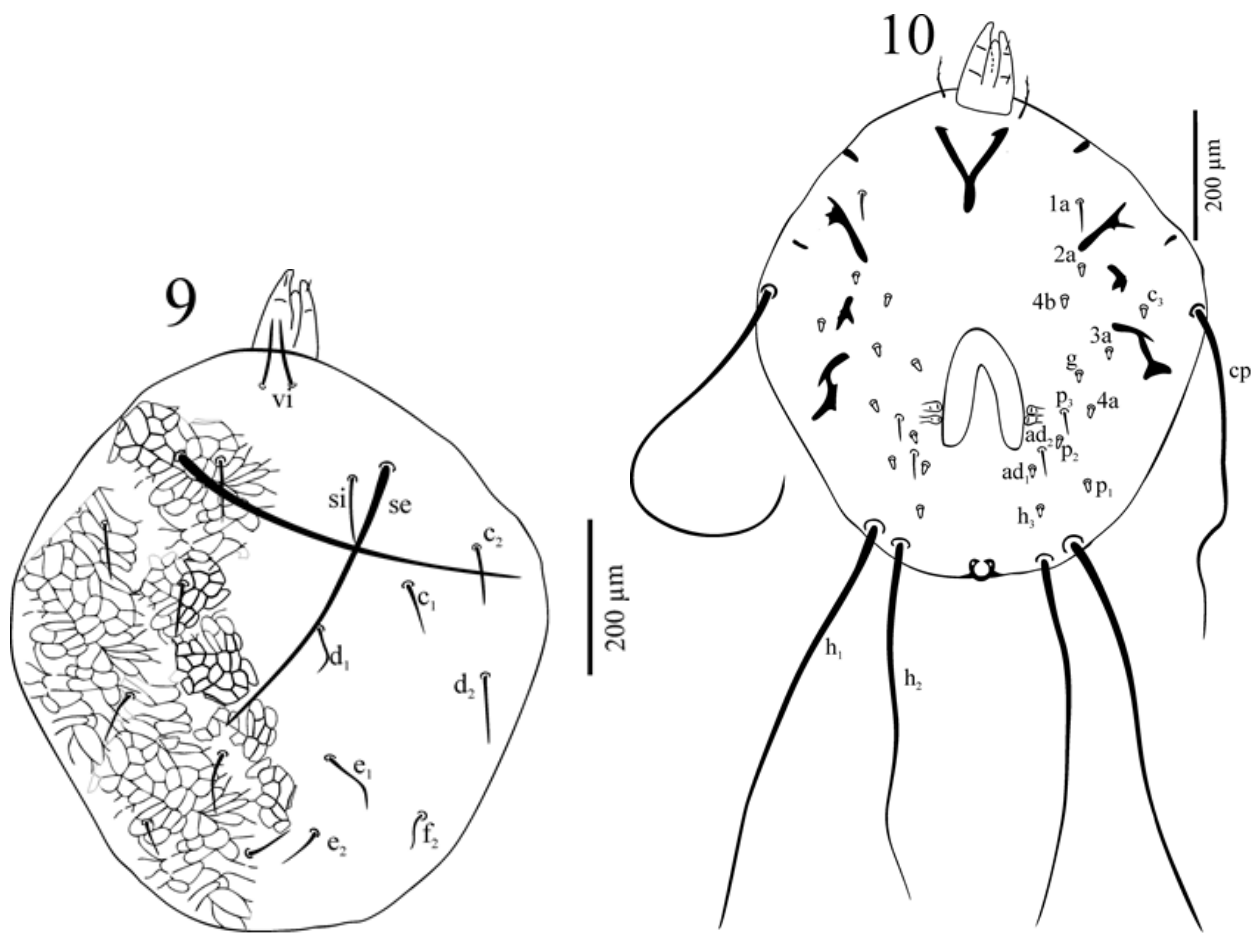

Figures 9-10. Cooremania kietaensis sp. nov. (female). 9, Dorsal view of idiosoma and gnathosoma, 10, Ventral view of idiosoma and gnathosoma.

Female $(n=2)$. The females have longer idiosoma than males, the dorsal setal pattern is identical as in males but all setae are longer, excluding setae h3 and $h_{2}$ (one male has this seta longer) (Fig. 9, Table 1).

The whole dorsum is ornamented. Ventral side of idiosoma differs from males in shape of setae in anal and genital region: setae $\mathrm{p}_{1}, \mathrm{p}_{2}, \mathrm{p}_{3}$ and $\mathrm{h}_{3}$ are peglike, in males tubercle-like (Fig. 10). Gnathosoma as in male, partly covered by idiosoma. 


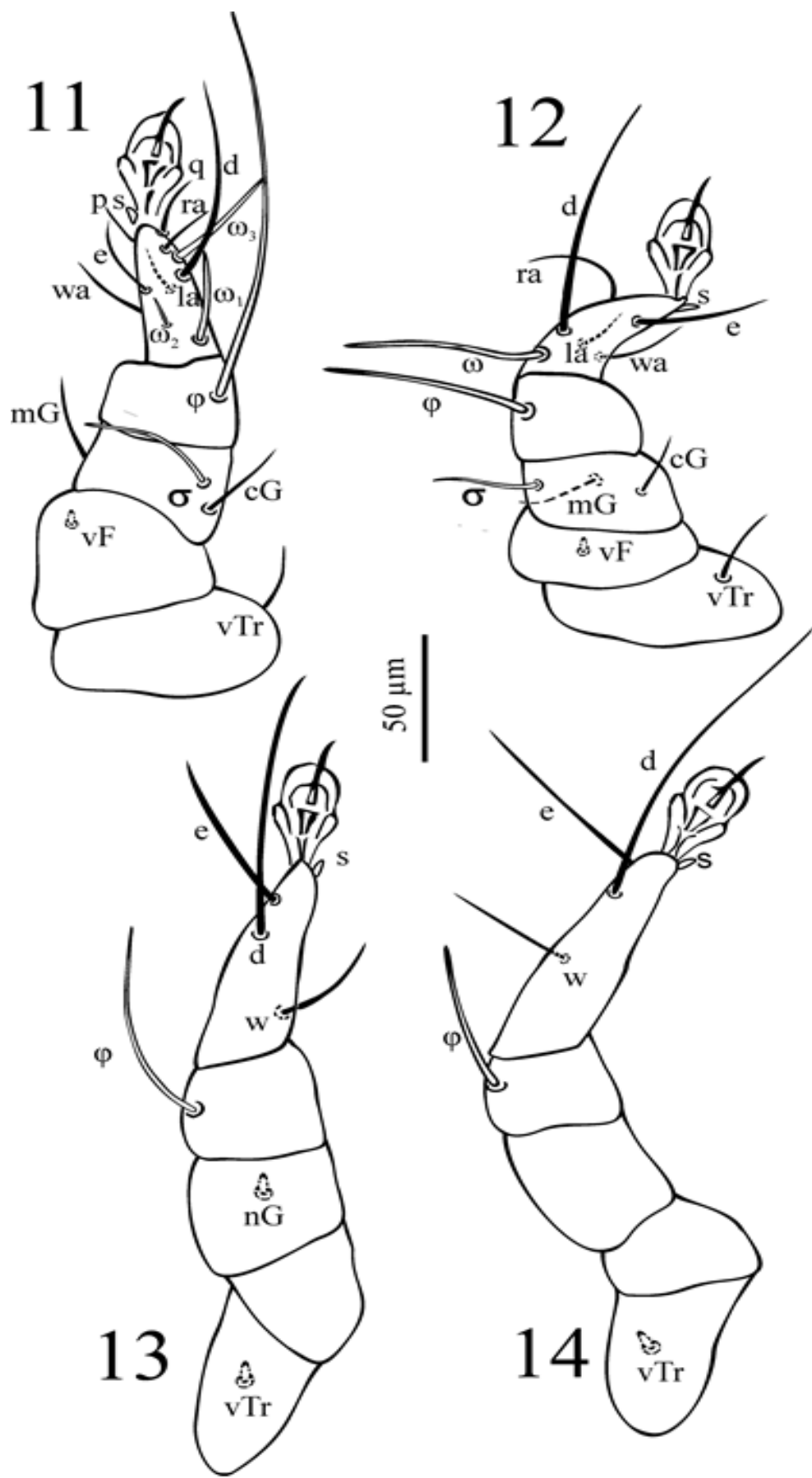

Figures 11-14. Cooremania kietaensis sp. nov. (female). 11, Leg I, 12, Leg II, 13, Leg III, 14. Leg IV. Leg setal formula. As in male; the only difference: seta vTr on leg IV is peg-like (Figs. 11-14). 

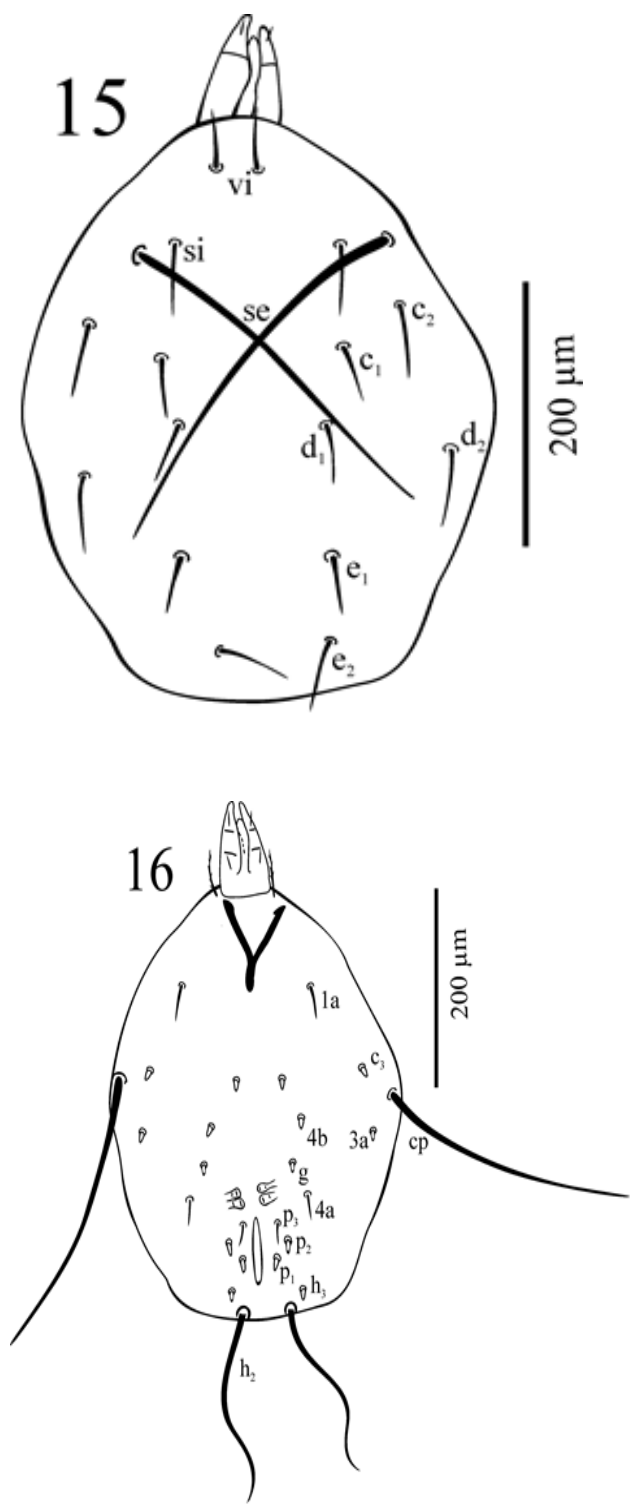

Figures 15-16. Cooremania kietaensis sp. nov. (protonymph). 15, Dorsal view of idiosoma and gnathosoma, 16, Ventral view of idiosoma and gnathosoma.

Protonymph $(\mathrm{n}=3)$. Propodosoma with three pairs of setae: long setae se and short setae vi and si. Hysterosoma with seven pairs of setae: long setae $h_{1}$ and short setae $c_{1}, c_{2}, d_{1}, d_{2}, e_{1}$ and $e_{2}$ (Fig. 15, Table 1) Ventral side of idiosoma with long setae $c p$ and $\mathrm{h}_{2}$ short and thin setae $1 \mathrm{a}, 4 \mathrm{a}$ and $\mathrm{p}_{3}$ and peg-like setae $\mathrm{c}_{3}, 3 \mathrm{a}, \mathrm{g}$, $4 b, p_{1}, p_{2}$ and $h_{3}$ (Fig. 16). Gnathosoma as in male, partly covered by idiosoma. 


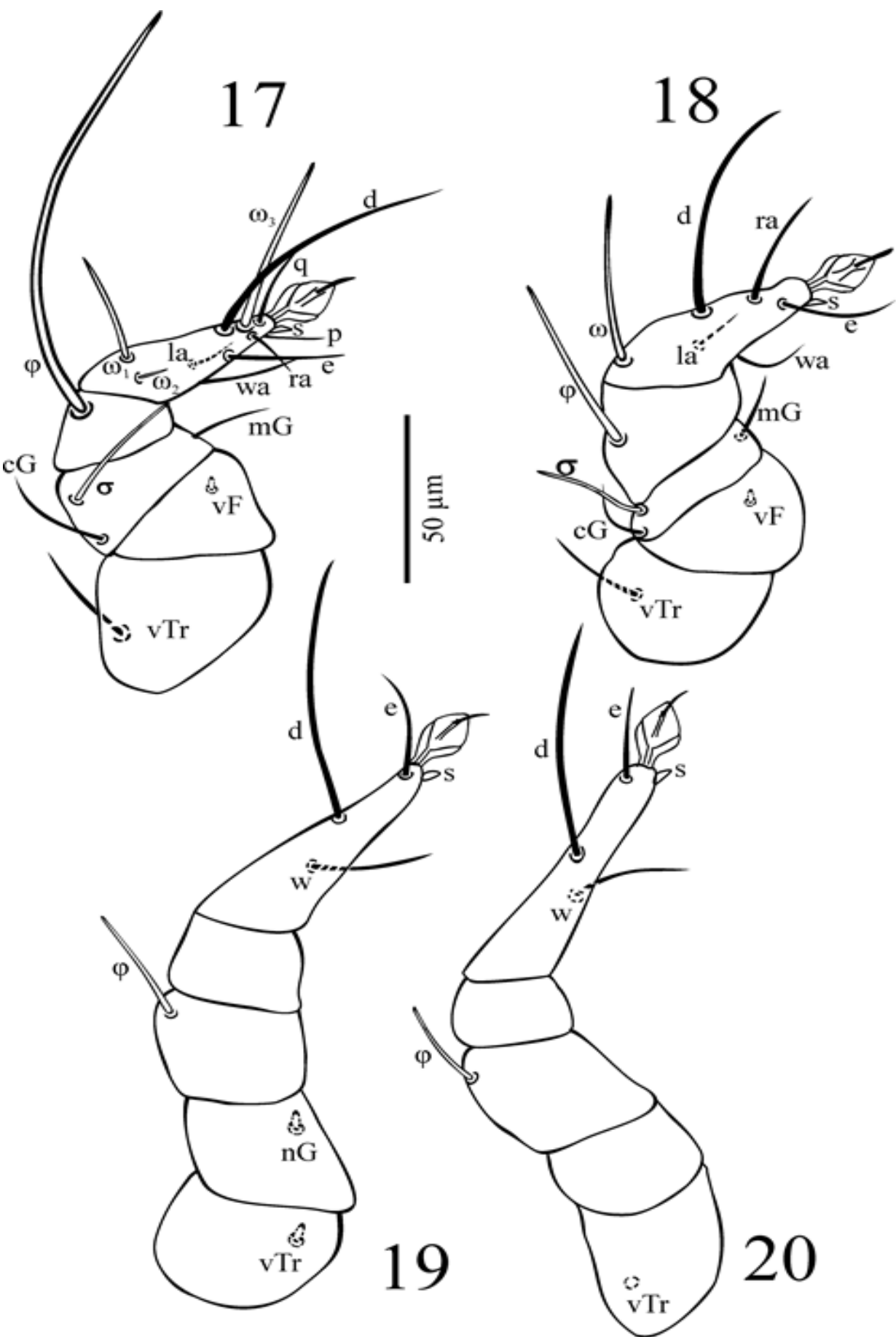

Figures 17-20. Cooremania kietaensis sp. nov. (protonymph). 17, Leg I; 18, Leg II; 19, Leg III, 20, Leg IV. 
Leg setal formula. Leg I: Ta $-\omega_{1}, \omega_{2}, \omega_{3}$, d, e, p, la, q, wa, s; Ti $-\varphi ; \mathrm{Ge}-$ $\sigma, \mathrm{mG}, \mathrm{cG} ; \mathrm{Fe}-\mathrm{vF} ; \mathrm{Tr}-\mathrm{vTr}$ (Fig. 17). Leg II: Ta $-\omega$, d, e, ra, wa, la, s; Ti - $\varphi$; $\mathrm{Ge}-\sigma, \mathrm{mG}, \mathrm{cG} ; \mathrm{Fe}-\mathrm{vF} ; \mathrm{Tr}-\mathrm{vTr}$ (Fig. 18). Leg III: Ta $-\mathrm{d}, \mathrm{e}, \mathrm{w}, \mathrm{s} ; \mathrm{Ti}-\varphi ; \mathrm{Ge}-$ nG; Tr - vTr (Fig. 19). Leg IV: Ta - d, e, w, s; Ti $\mathrm{vF}$ on Fe I and Fe II and $\mathrm{nG}$ on Ge III nd vTr on Tr III are peg-like.

Measurements are given in Table 1.

Etymology -Named after the type locality.

Type material -Holotype male, seven paratypes: two males and two females and three protonymphs, Kieta, date undetermined, Bougainville Island, Papua New Guinea from undetermined Lucanidae (Insecta; Coleoptera); collector undetermined. All mites are deposited in the Museum of Natural History, Wrocław University, Poland.

Remarks. Cooremania kietaensis sp. nov. differs from C. wauensis in males in longer tubercle-like spines with dumpy bases $\mathrm{p}_{1}, \mathrm{p}_{2}, \mathrm{p}_{3}, \mathrm{~h}_{3}>23 \mu \mathrm{m}$ vs. vs. these setae are peg-like, $<15 \mu \mathrm{m}$, setae $\mathrm{h} 1$ and se shorter than idiosoma vs. setae $\mathrm{h} 1$ and se longer than idiosoma, longer solenidion $\varphi$ on Ti I (over two times longer than Ta I) vs. solenidion $\varphi$ on Ti I slightly longer than Ta I, peg-like seta on genu III vs. tubercle-like spine and seven pairs of short setae on hysterosoma vs. six pairs of setae on hysterosoma; in females in posterior margin of idiosoma slightly concave vs. posterior margin almost straight, setae se and h1 shorter than idiosoma vs. setae se and h1 longer than idiosoma, solenidion $\varphi$ on Ti I over two times longer than Ta I vs. solenidion $\varphi$ on Ti I shorter than Ta I and hysterosoma with seven pairs of short setae vs. hysterosoma with six pairs of short setae; protonymphs can be not compare on account of laconic Nesbitt description.

\section{REFERENCES}

Berlese, A. (1881): Sopra un nuovo generi di Acari parassiti degli insetti. Atti del Reale Istituto Veneto di Scienze, lettere ed arti 7, 747-752.

Bolu, H. (2016): Southeastern Anatolia region insect fauna I (Coleoptera I: Caraboidea; Dytiscoidea; Bostrichoidea; Chrysomeloidea; Cleroidea; Cucujoidea) of Turkey, Agriculture \& Forestry, Vol. 62 Issue 4: 125-145, 2016.

Grandjean, F. (1939): La chaetotaxie des pattes chez les Acaridiae. Bulletin de la Societé, Zoologique de France, 64, 50-56.

Griffiths, D. A., Atyeo, W., Norton, R. A., Lynch, C. A. (1990): The idiosomal chaetotaxy of astigmatid mites. Journal of Zoology (London), 220, 1-32.

Haitlinger, R. (1989a): New canestriniid mites (Acari, Astigmata, Canestriniidae) connected with beetles of the subfamilies Dynastinae and Cetoniinae (Insecta, Coleoptera, Scarabaeidae). Wiadomości Parazytologiczne, 35, 4360.

Haitlinger, R. (1989b): New canestriniid mites (Acari, Astigmata, Canestriniidae) associated with Scarabaeidae and Cerambycidae (Insecta, Coleoptera) from the Indo-Australian Region. Wiadomości Parazytologiczne, 35, 585-601. 
Haitlinger, R. (1990a): New canestriniid mites (Acari, Astigmata, Canestriniidae) associated with beetles of the families Carabidae, Scarabaeidae, Tenebrionidae and

Passandridae (Insecta, Coleoptera). Annales Zoologici, 43, 111-125.

Haitlinger, R. (1990b): New canestriniid mites (Acari, Astigmata, Canestriniidae) associated with passalid beetles (Insecta, Coleoptera, Passalidae). Polskie Pismo Entomologiczne, 59, 527-589.

Haitlinger, R. (1991): Rugoniphela n. gen., Noemiphela n. gen. and Uriophela $\mathrm{n}$. gen. three new genera of canestriniid mites (Acari, Astigmata) from Asia. Redia, 74, 534-542.

Haitlinger, R. (1993): New genus and two new species of canestriniid mites (Acari, Astigmata) associated with Lucanidae (Insecta, Coleoptera). Mit Zool Mus Berl 69: 339-344.

Haitlinger, R. (1995): New species of mites collected on Tenebrionidae, Scarabaeidae,

Cerambycidae and Lucanidae with the description of new subfamily Vereoxiinae

(Acari, Astigmata, Canestriniidae). Wiadomości Parazytologiczne, 41, 7991.

Haitlinger, R., Šundić, M. (2016): Ibizella balearica n. gen., n. sp. (Astigmata

Canestriniidae) from Balearic Islands, Spain. Redia, XCIX, 75-82. http://dx.doi.org/10.19263/REDIA-99.16.09

Kim, W., Lee, S., Choi, J., Sim, H., Kim, J. (2006): A new canestriniid mite, Haitlingeria longilobata n. g., n. sp. (Acari: Astigmata: Canestriniidae), parasitizing Serodnathus platymelus castanicolor Motschulsky (Coleoptera: Lucanidae) from South Korea. Systematic Parasitology, 65, 13-18. DOI: $10.1007 / \mathrm{s} 11230-006-9035-9$

Kishida, K. (1924): On a new canestriniid mite from Japan. Annot Zool Jap 10: 363-364.

Nesbitt, H. (1976): Five new canestriniids (Acari: Astigmata) from Papau New Guinea. Zoological Journal of the Linnean Society, 58, 219-236.

Norton, R. A. (1998): Morphological evidence for the evolutionary origin of Astigmata

(Acari: Acariformes). Experimental \& Applied Acarology, 22, 559-594. DOI: 10.1023/a:1006135509248

Okabe, K., Goka, K. (2008): Potential impacts on Japanese fauna of canestriniid mites (Acari: Astigmata) accidentally introduced with pet lucanid beetles Southeast Asia. Biodiversity and Conservation, 17, 71-81. DOI: 10.1007/s10531-007-9231 1

Summers, F. M., Schuster, R. O. (1981a): Apalotacaraus, a new genus of canestriniid mites from passalid beetles. International Journal of Acarology, 7, 17-38. 
Summers, F. M., Schuster, R. O. (1981b): Three new genera of canestriniid mites associated with passalid beetles. International Journal of Acarology, 7, 3946.

Trach, V. A., Khaustov, A. A. (2011) A review of the genus Coleopterophagus Berlese, 1882 (Acari: Astigmata: Canestriniidae) of Ukraine. Acarina, 19, 213-230. 